\title{
Quantification of Unimolecular Photoreaction Kinetics: Determination of Quantum Yields and Development of Actinometers-The Photodegradation Case of Cardiovascular Drug Nisoldipine
}

\author{
Mounir Maafi and Wassila Maafi \\ Leicester School of Pharmacy, De Montfort University, The Gateway, Leicester LE1 9BH, UK \\ Correspondence should be addressed to Mounir Maafi; mmaafi@dmu.ac.uk
}

Received 9 March 2015; Accepted 13 May 2015

Academic Editor: Adel A. Ismail

Copyright (C) 2015 M. Maafi and W. Maafi. This is an open access article distributed under the Creative Commons Attribution License, which permits unrestricted use, distribution, and reproduction in any medium, provided the original work is properly cited.

\begin{abstract}
The lack of integrated rate-laws for photoreactions has led to carry out the treatment of drugs photodegradation kinetic data using the classical zeroth-, first-, and second-order kinetics that were originally developed for thermal reactions. The recent developments of $\Phi$-order kinetic models has opened new perspectives in the treatment of photoreaction kinetics of systems involving a photolabile molecule (A) transforming into a photochemically and thermally stable product (B), that is, the $\mathrm{AB}(1 \Phi)$ photoreaction systems. Within this framework, the kinetics of cardiovascular and photosensitive drug nisoldipine (NIS) has been rationalised. Continuous and monochromatic irradiation of NIS in ethanol obeyed $\Phi$-order kinetics with a sigmoid-shaped quantum yield variation with irradiation wavelength (0.0041-0.35 within 235-390 nm spectral region). Both NIS initial concentration-induced self-photostabilisation effect and the photostabilisation by absorption competitors were quantified (up 70\%) and related to a reduction of the photokinetic factor. Finally, the $\Phi$-order kinetics also served to demonstrate the actinometric potential of NIS for the 320-400 nm dynamic range.
\end{abstract}

\section{Introduction}

$\Phi$-order kinetics is a recently developed approach useful for the investigation and characterisation of unimolecular and reversible photodegradation reactions of drugs [1-7]. It has been proven to be more suitable for the description of drugs' photoreactivity than the classical treatments based on zeroth, first, and second reaction orders [1-4]. In this respect, $\Phi$-order kinetics palliate the main drawbacks of the classical strategy in four aspects: (i) its equations are based on the differential equations of photo and not thermal reactions, (ii) it allows achieving a unique interpretation of the experimental data of reaction kinetic profiles conversely to the thermal strategy that may lead to ambiguity on the true order obeyed by the photoreaction studied, (iii) it applies to the whole set of data making up the kinetic trace of the phototransformation and is not limited to a partial section (usually up to the half-life time) of the trace, and (iv) it provides an analytical expression for the reaction rate-constant that refines the physical meaning of this parameter for such photoreactions. As such, $\Phi$-order kinetics may serve to amend the ICH recommendations by introducing procedures and standard kinetic data treatment methods for the quantification of drugs' photodegradation kinetics that are lacking in the Q1b document relative to the photostability of drugs [8].

Nisoldipine (NIS) (( \pm )3-isobutyl-5-methyl-1,4-dihydro2,6-dimethyl-4-(2-nitrophenyl)-pyridine-3,5-dicarboxylate) is a member of the 1,4-dihydropyridine class of calcium channel antagonist drugs. NIS is prescribed for the treatment of hypertension and angina [9].

The presence of a nitro group at the ortho-position of the phenyl ring in some members of the 1,4-dihydropyridines such as NIS and nifedipine makes them particularly prone to photolability. The photodegradation mechanism of NIS 


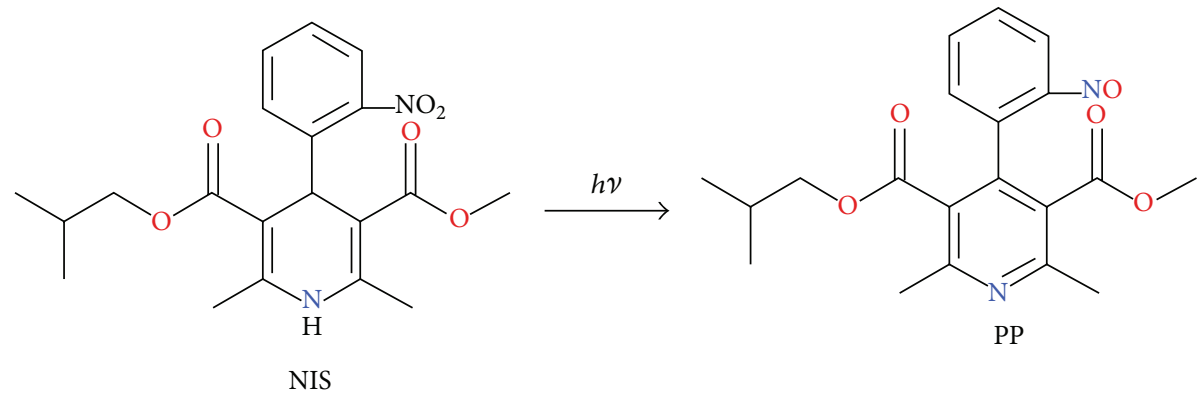

SCHEME 1: Nisoldipine (NIS) unimolecular and light induced transformation.

(Scheme 1) involves an oxidation of the dihydropyridine moiety to a pyridine ring and the reduction of the aromatic nitro group to a nitroso [9]. This change in chemical structure results in a significant loss of therapeutic efficacy [10]. As a consequence, a number of studies have been devoted to the study of the photodegradation of NIS under various conditions.

Although much effort has been devoted to the elucidation of the photodecomposition products of NIS, studies on the kinetics of its photodegradation remain scarce and largely controversial. For instance, the photoconversion of NIS in methanolic solutions using UV spectrophotometry and RP-HPLC was attributed a first-order kinetics [11], whereas another similar spectrophotometric study in ethanol described NIS kinetics by an apparent first-order up to a concentration of $3 \times 10^{-5} \mathrm{M}$ and the zero-order for concentrations higher than $2 \times 10^{-4} \mathrm{M}$ [12]. In the solid state, NIS photodegradation monitored by HPLC was ascribed a zeroorder kinetics under daylight but the reaction order could not be clearly identified under UV illumination. In the latter case, NIS degradation showed a more complicated kinetic behaviour whereby the decrease of its concentration was linear with time up to $c a$. $60 \%$ of the initial concentration then it followed a much slower regime [13]. However, the primary photoprocess of NIS degradation in solution leads to a unique photoproduct: the nitroso derivative, PP in Scheme 1 $[9,14,15]$, and any minor products that might be detected in the reactive medium [10] must result from further slow thermal degradation of PP [14].

The aim of this paper is to conduct a full elucidation of NIS kinetics in ethanol using $\Phi$-order kinetics. The irradiation-dependent quantum yields will be determined and the effects of initial concentration and absorption competitors quantified. Finally, the potential use of NIS in actinometry will be demonstrated.

\section{Materials and Methods}

2.1. Materials. Nisoldipine (NIS) (( \pm )3-isobutyl-5-methyl-1,4dihydro-2,6-dimethyl-4-(2-nitrophenyl)-pyridine-3,5-dicarboxylate), tartrazine (TRZ), quinoline yellow (QY), and spectroscopic grade ethanol were purchased from Aldrich and were used without further purification.

2.2. Monochromatic Continuous Irradiation. For irradiation experiments, a Ushio $1000 \mathrm{~W}$ xenon arc-lamp light source housed in a housing shell model A6000 and powered by a power supply model LPS-1200 was used. This setting was cooled by tap water circulation through a pipe system. The lamp housing was connected to a monochromator model 101 that allows the selection of specific irradiation wavelengths since it consists of a special $\mathrm{f} / 2.5$ monochromator with a 1200 groove/mm at $300 \mathrm{~nm}$ blaze grating. The excitation beam was guided through an optical fibre to impinge from the top of the sample cuvette; that is, the excitation and the analysis light beams were perpendicular to each other. The setup was manufactured by Photon Technology International Corporation.

2.3. The Monitoring System. A diode array spectrophotometer (Agilent 8453) was used to measure the various absorption spectra and kinetic profiles for the irradiation and calibration experiments. This spectrophotometer was equipped with a $1 \mathrm{~cm}$ cuvette sample holder and a Peltier system model Agilent 8453 for temperature control. As such, the sample was kept at $22^{\circ} \mathrm{C}$, stirred continuously during the experiment, and completely shielded from ambient light. The spectrophotometer was monitored by an Agilent 8453 Chemstation kineticssoftware.

A Radiant Power/Energy meter model 70260 was used to measure the radiant power of the incident excitation beams.

2.4. Kinetic Data Treatment. In order to carry out nonlinear fittings and to determine best-fit curves, a LevenbergMarquardt iterative program within the Origin 6.0 software was used.

2.5. Solutions. A $4.9 \times 10^{-4} \mathrm{M}$ stock solution of NIS in ethanol was prepared by weighing the solid. The stock solution was diluted to prepare fresh analytical solutions $\left(\mathrm{ca} .7 .8 \times 10^{-6} \mathrm{M}\right)$ for analysis of irradiation experiments performed at various wavelengths.

Since NIS absorbs in the UV and visible regions of the spectrum, particular care was taken during the handling of NIS solutions using aluminium foil paper wrapping and keeping them in the fridge while not in use. As such, NIS stock solutions remained stable for several weeks.

Stock solutions of TRZ $\left(9.14 \times 10^{-5} \mathrm{M}\right)$ and QY $(6.17 \times$ $10^{-5} \mathrm{M}$ ) were prepared in ethanol from their respective powders. Diluted solutions of the dyes in ethanol were then spiked with volumes from NIS stock to make up solutions of the required concentrations. 
TRZ and QY were selected since their spectrum largely overlaps that of NIS and could therefore act as competitive absorbers of irradiation. Photoprotection experiments were conducted in quartz cuvettes. The spectrum of the dye solution (of known concentration) was taken before this solution was used for a blank experiment. Subsequently, a specific volume (a few $\mu \mathrm{L}$ ) of NIS stock solution was added to the dye solution. Thus, it was possible to record only the UV/Vis spectra of the reactive medium (NIS and PP) during the reaction progress (with the measurement not being hampered by the absorbance of the dye, present in the medium).

For actinometric studies, NIS ethanolic solutions of the same concentrations $\left(c a .7 \times 10^{-6} \mathrm{M}\right)$ were exposed to specific irradiation wavelengths using a series of different intensities for each wavelength. The kinetic traces were observed at an observation wavelength of $390 \mathrm{~nm}$ and were subsequently fitted with the $\Phi$-order equations.

Experiments were conducted at least in triplicates.

\section{Theoretical Basis}

3.1. The Ф-Order Semiempirical Integrated Rate-Law for $A B(1 \Phi)$ Systems. The general semiempirical integrated ratelaw equation describing the time variation of the cumulative absorbance of the reactive medium involving the phototransformation of NIS into its photoproduct (PP) (Scheme 1) is given by the logarithmic equation (1) [3]. This type of reactions involving a single photochemical step $\left(\Phi_{\mathrm{A} \rightarrow \mathrm{B}}^{\lambda_{\text {ir }}}\right)$ between the initial species (A) and its photoproduct (B) are generally labelled $\mathrm{AB}(1 \Phi)$ systems. The formula of (1) has been established considering that the solution, subjected to a monochromatic, nonisosbestic, and continuous irradiation $\left(\lambda_{\text {irr }}\right)$, is homogeneously and continuously stirred. It is also assumed that the concentration of the excited-state species is negligible, the medium temperature is constant, and, at the irradiation wavelength $\left(\lambda_{\text {irr }}\right)$, species NIS and PP may absorb different amounts of light $\left(P_{\lambda_{\text {irr }}}\right)$; that is, the absorption coefficients of the species may be different $\left(\varepsilon_{\mathrm{NIS}}^{\lambda_{\text {irr }}} \neq \varepsilon_{\mathrm{PP}}^{\lambda_{\text {irr }}} \neq 0\right)$ :

$$
\begin{aligned}
& A_{\text {tot }}^{\lambda_{\text {irt }} / \lambda_{\text {obs }}}(t)=A_{\mathrm{PP}}^{\lambda_{\text {irf }} / \lambda_{\text {obs }}}(\infty) \\
& +\frac{A_{\mathrm{NS}}^{\lambda_{\mathrm{irr}} / \lambda_{\text {obs }}}(0)-A_{\mathrm{PP}}^{\lambda_{\text {irr }} / \lambda_{\text {obs }}}(\infty)}{A_{\mathrm{NIS}}^{\lambda_{\text {irr }} / \lambda_{\text {irr }}}(0)-A_{\mathrm{PP}}^{\lambda_{\text {irr }} / \lambda_{\text {irr }}}(\infty)} \times \frac{l_{\lambda_{\text {obs }}}}{l_{\lambda_{\text {irr }}}} \times \log [1 \\
& +\left(10^{\left[\left(A_{\mathrm{NIS}}^{\lambda_{\mathrm{irr}} / \lambda_{\mathrm{irr}}}(0)-A_{\mathrm{PP}}^{\lambda_{\mathrm{irr}} / \lambda_{\mathrm{irr}}}(\infty)\right) \times\left(l_{\lambda_{\mathrm{irr}}} / l_{\lambda_{\mathrm{obs}}}\right)\right]}-1\right) \\
& \left.\times e^{-k_{\mathrm{NIS}}^{\lambda_{\mathrm{irr}}} \times t}\right]
\end{aligned}
$$

with $l_{\lambda_{\text {irr }}}$ being the optical path length of the irradiation beam inside the sample and $l_{\lambda_{\text {obs }}}$ is the optical path length of the spectrophotometer monitoring light. The absorbances $\left(A^{\lambda / \lambda}\right)$ in (1) correspond to the observed measurements by the instrument (i.e., along $l_{\lambda_{\text {obs }}}$ ) of the reactive medium, at a given reaction time $\left(A_{\text {tot }}^{\lambda_{\text {tir }} /} / \lambda_{\text {obs }}(t)\right)$, at the initial time $(t=$ $0, A_{\mathrm{NIS}}^{\lambda_{\text {irr }} / \lambda_{\text {obs }}}(0)$ and $\left.A_{\mathrm{NIS}}^{\lambda_{\text {irr }} / \lambda_{\text {irr }}}(0)\right)$, and at the end of the reaction $\left(t=\infty, A_{\mathrm{PP}}^{\lambda_{\mathrm{irr}} / \lambda_{\mathrm{obs}}}(\infty)\right.$ and $A_{\mathrm{PP}}^{\lambda_{\mathrm{irr}} / \lambda_{\text {irr }}}(\infty)$ where only the photoproduct is present). The absorbances can be read out from the spectra at the particular wavelengths of irradiation $\left(\lambda_{\text {irr }}\right)$ and observation $\left(\lambda_{\text {obs }}\right)$ which might or not be the same. Therefore, each absorbance is dually labelled by the irradiation and the observation wavelengths $\left(\lambda_{\text {irr }} / \lambda_{\text {irr }}\right.$ or $\lambda_{\text {irr }} / \lambda_{\text {obs }}$ ) corresponding to the specific measurement.

The exponential factor in (1), $k_{\mathrm{NIS}}^{\lambda_{\text {irr }}}$, represents the overall photoreaction rate-constant, which can be analytically expressed as

$$
\begin{aligned}
k_{\mathrm{NIS}}^{\lambda_{\mathrm{irr}}} & =\Phi_{\mathrm{NIS} \rightarrow \mathrm{PP}}^{\lambda_{\mathrm{irr}}} \times \varepsilon_{\mathrm{NIS}}^{\lambda_{\mathrm{irr}}} \times l_{\lambda_{\mathrm{irr}}} \times F_{\mathrm{D}}^{\lambda_{\mathrm{irr}}} \times P_{\lambda_{\mathrm{irr}}} \\
& =\beta_{\lambda_{\mathrm{irr}}} \times P_{\lambda_{\mathrm{irr}}},
\end{aligned}
$$

where $\Phi_{\mathrm{NIS}}^{\lambda_{\mathrm{irr}}} \rightarrow$ PP is the quantum yield of NIS photoconversion, realised at the nonisosbestic irradiation wavelength $\left(\lambda_{\text {irr }}\right)$, $\varepsilon_{\text {NIS }}^{\lambda_{\text {irr }}}$ is the molar absorption coefficient of NIS, $P_{\lambda_{\text {irr }}}$ is the radiant power, and $F_{\infty}^{\lambda_{\text {irr }}}$ the photokinetic factor expressed as

$$
F_{\infty}^{\lambda_{\text {irr }}}=\frac{1-10^{-\left(A_{\mathrm{tot}}^{\lambda_{\mathrm{irr}} / \lambda_{\mathrm{irr}}}(\infty) \times\left(l_{\mathrm{irr}} / l_{\mathrm{obs}}\right)\right)}}{A_{\mathrm{tot}}^{\lambda_{\mathrm{irr}} / \lambda_{\mathrm{irr}}}(\infty) \times\left(l_{\lambda_{\mathrm{irr}}} / l_{\lambda_{\mathrm{obs}}}\right)} .
$$

It is worth noting that in the case where another absorbing species is present in the reactive medium with NIS without being itself photoactive, such as a pharmaceutical excipient; the formula of the photokinetic factor (3) must be amended to take into account the effect of such an exogenous absorbing species. This is implemented by considering that the total absorbance of the medium at reaction completion includes both PP $\left(A_{\mathrm{PP}}^{\lambda_{\text {irr }} / \lambda_{\text {irr }}}(\infty)\right)$ and excipient $\left(A_{\text {Excip. }}^{\lambda_{\text {irr }} / \lambda_{\text {irr }}}\right)$ absorbances; that is, $A_{\text {tot }}^{\lambda_{\text {irr }} / \lambda_{\text {irr }}}(\infty)=A_{\mathrm{PP}}^{\lambda_{\text {irr }} / \lambda_{\text {irr }}}(\infty)+A_{\text {Excip }}^{\lambda_{\text {irr }} / \lambda_{\text {irr }}}$, instead of being only equal to that of PP, $A_{\text {tot }}^{\lambda_{\text {irt }} / \lambda_{\text {irr }}}(\infty)=A_{\mathrm{PP}}^{\lambda_{\text {irr }} / \lambda_{\text {irr }}}(\infty)$, as is the case in the absence of excipient [3].

The aforementioned equations (1)-(3) have been validated by testing their reliability to fit synthetic data for $\mathrm{AB}(1 \Phi)$ systems that have been generated by Runge-Kutta numerical integration method. The optimization of these equations was realised by considering a large number of reactions including a wide range of reaction attributes and experimental conditions [3]. The development of this semiempirical framework was meant to circumvent the impossibility to achieve a closed-form integration of the photoreaction differential equation when both initial species and photoproduct absorb. The expression, previously established for the photodegradation of the initial species as the only absorbing species $\left(\varepsilon_{B}^{\lambda_{\text {irr }}}=0\right)[1]$, represented a template for the derivation of (1). In fact, the formulae for the latter case can be retrieved from (1)-(3) by considering the quantities relative to the photoproduct as equal to zero (i.e., $A_{\mathrm{PP}}^{\lambda_{\mathrm{irr}} / \lambda_{\mathrm{irr}}}(\infty)=$ $A_{\mathrm{PP}}^{\lambda_{\text {ir }} / \lambda_{\text {obs }}}(\infty)=\varepsilon_{\mathrm{PP}}^{\lambda_{\text {irr }}}=0$ ). Therefore, (1)-(3) can be considered as universal formulae applicable to the photodegradation of any $\mathrm{AB}(1 \Phi)$ system at any nonisosbestic monochromatic irradiation wavelength irrespective of whether the photoproduct absorbs at that irradiation wavelength.

Nevertheless, the application of (1)-(3) was conditional to $F_{\mathrm{\infty}} \lambda_{\text {irr }}$ being higher than 1.2 (corresponding to $A_{\mathrm{PP}}^{\lambda_{\text {irr }} / \lambda_{\text {irr }}}(\infty)<$ 
0.65) for the cases where both reactive species and photoproduct absorb, but in the absence of an excipient. This has proven to be a simple limitation as the former condition can easily be met by lowering the concentration of the initial species or possibly by reducing the irradiation path length $\left(l_{\lambda_{\text {irr }}}\right)$. In the presence of an exogenous excipient molecule, the condition on PP $\left(A_{\mathrm{PP}}^{\lambda_{\text {irr }} / \lambda_{\text {irr }}}(\infty)<0.65\right)$ holds with an additional limit on the highest absorbance that can be used for the excipient at the irradiation wavelength; $A_{\text {Excip. }}^{\lambda_{\text {irr }}}<2.5$, a value that is generally beyond the linearity range of most organic molecules [3].

The formulation of the photokinetic factor (3) indicates that its value decreases with increasing total absorbance of the medium at the irradiation wavelength. As a consequence, the increase of $A_{\text {tot }}^{\lambda_{\text {irr }} / \lambda_{\text {irr }}}(\infty)$ induces a decrease in the overall photodegradation rate-constant.

Another important parameter that may find use in the determination of quantum yields and in actinometry is the rate-law for $\mathrm{AB}(1 \Phi)$ photoreactions at $t=0$, namely, the initial reaction velocity, expressed as

$$
\begin{aligned}
v_{0}^{\lambda_{\text {irr }} / \lambda_{\text {obs }}=} & \left(\varepsilon_{\mathrm{PP}}^{\lambda_{\text {obs }}}-\varepsilon_{\mathrm{NIS}}^{\lambda_{\text {obs }}}\right) \times l_{\lambda_{\text {obs }}} \times \Phi_{\mathrm{NIS}}^{\lambda_{\text {irr }}} \times \mathrm{PP}_{\mathrm{NIS}}^{\lambda_{\text {irr }}} \\
& \times l_{\lambda_{\text {irr }}} \times F_{0}^{\lambda_{\text {irr }}} \times C_{0} \times P_{\lambda_{\text {irr }}}=\delta_{\lambda_{\text {irr }}} \times P_{\lambda_{\text {irr }}}
\end{aligned}
$$

with $F_{0}^{\lambda_{\text {irr }}}$ being the photokinetic factor at $t=0$ and $\delta_{\lambda_{\text {irr }}}$ a proportionality factor.

\subsection{Integrated Rate-Law Equations for Isosbestic Irradiation.} The equations presented above were set for nonisosbestic irradiation exclusively because the semiempirical modelling was based on a formula derived for nonisosbestic irradiation. Also, because the semiempirical equation (1) has not been derived by a closed-form integration, its formulation is incomplete and does not apply for all situations as suggested by the limitations on the absorbance values of the medium (e.g., $\left.F_{\infty}^{\lambda_{\text {irr }}}<1.2\right)$. This is also evident for isosbestic irradiation $\left(\lambda_{\text {isos }}\right)$ as the logarithmic formula (1) is not at all defined for isosbestic conditions (since the denominator $A_{\text {NIS }}^{\lambda_{\text {isos }} / \lambda_{\text {isos }}}(0)-$ $\left.A_{\mathrm{PP}}^{\lambda_{\text {isos }} / \lambda_{\text {isos }}}(\infty)=0\right)$. Therefore, a set of equations needs to be derived for this situation even though only a few isosbestic points usually exist on the spectra.

The invariance of the absorbance at the irradiation wavelength at an isosbestic irradiation, leading to a constant photokinetic factor throughout the reaction time, renders integration of this system's differential equation possible [16]. Hence, the kinetics obey a monoexponential integrated ratelaw, given by

$$
\begin{aligned}
A_{\mathrm{tot}}^{\lambda_{\text {isos }} / \lambda_{\mathrm{obs}}}(t)= & A_{\mathrm{tot}}^{\lambda_{\mathrm{isos}} / \lambda_{\mathrm{obs}}}(0) \\
& +\left(A_{\mathrm{tot}}^{\lambda_{\mathrm{isos}} / \lambda_{\mathrm{obs}}}(0)-A_{\mathrm{tot}}^{\lambda_{\mathrm{isos}} / \lambda_{\mathrm{obs}}}(\infty)\right) \\
& \times\left(e^{-k_{\mathrm{NIS}}^{\lambda_{\mathrm{isos}}} \times t}-1\right)
\end{aligned}
$$

with its overall reaction rate-constant expressed as

$$
k_{\mathrm{NIS}, \mathrm{PP}}^{\lambda_{\text {isos }}}=\Phi_{\mathrm{NIS} \rightarrow \mathrm{PP}}^{\lambda_{\text {isos }}} \times \varepsilon_{\mathrm{NIS}}^{\lambda_{\text {isos }}} \times l_{\lambda_{\text {isos }}} \times P_{\lambda_{\text {isos }}} \times F_{\lambda_{\text {isos }}} .
$$

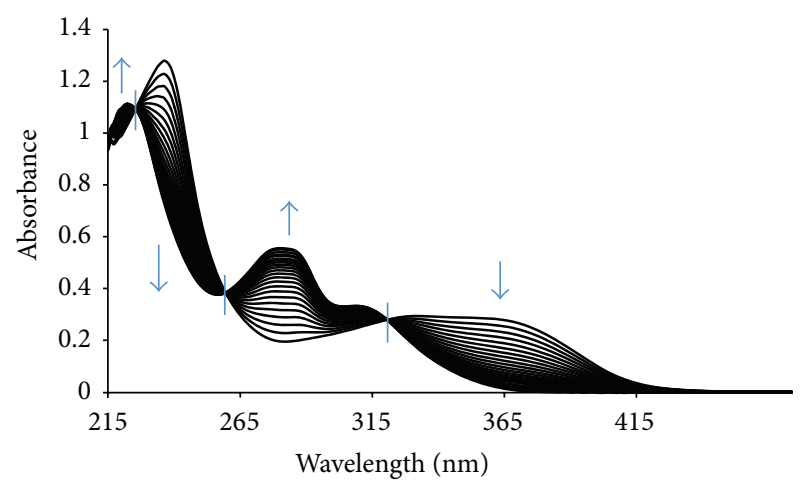

FIGURE 1: Evolution of the electronic absorption spectra of $5.88 \times$ $10^{-5} \mathrm{M}$ NIS in ethanol subjected to a continuous irradiation with a $390 \mathrm{~nm}$ monochromatic beam (total irradiation time $2000 \mathrm{~s}$ at a radiant power of $P_{390}=7.76 \times 10^{-7}$ einstein. $\mathrm{s}^{-1} \cdot \mathrm{dm}^{-3}$ ). The arrows indicate the direction of the peaks' evolution during the photoreaction and the vertical lines cross the spectra at the isosbestic points $(232,259$, and $320 \mathrm{~nm})$.

The reaction initial velocity for isosbestic conditions has the same formula as (4) but with $\lambda_{\text {irr }}$ being replaced by $\lambda_{\text {isos }}$.

\section{Results and Discussion}

4.1. Photodegradation of NIS. The absorbance spectrum of NIS in ethanol is characterised by two main absorption 200250 and 300-420 $\mathrm{nm}$ regions, spanning the UVB, UVA, and part of the visible spectral ranges (Figure 1). The main peak maxima for NIS in ethanol are located at 238 and $340 \mathrm{~nm}$ with the former having a much higher absorbance than the latter broader peak. NIS spectrum may be interpreted by the contribution of the independent absorptions of the nitrobenzene and the dihydropyridine chromophores. The long wavelength band (300-420 nm) belongs exclusively to the latter molecular moiety, whereas both chromophores input the UVB transition [17].

The photodegradation of NIS is easily evidenced by a change in the UV spectrum of the solution (Figure 1). Exposure of ethanolic solutions of NIS to monochromatic irradiation results in the gradual increase of the absorbance regions of 200-230 $\mathrm{nm}$ and 260-320 $\mathrm{nm}$ with a peak appearing in the latter region at 280 and a shoulder at $314 \mathrm{~nm}$. A decrease in absorbance is also observed between 230 and $260 \mathrm{~nm}$ and $320-420 \mathrm{~nm}$, with the appearance of three isosbestic points at 232, 259, and $320 \mathrm{~nm}$. The region between 390 and $420 \mathrm{~nm}$ represents an exclusive absorption region for NIS as the spectrum of PP ends at $c a .370 \mathrm{~nm}$. The timely evolution of the spectra and the simultaneous formation of identifiable isosbestic points suggest that the photoreaction proceeds smoothly without formation of by-products. Moreover, the irradiation of the PP (that was formed from NIS) at various wavelengths did not result in further spectral changes, indicating that the PP is photostable.

The hypsochromic shift observed in the absorption range $320-420 \mathrm{~nm}$ has been assigned to the $\pi \rightarrow \pi^{*}$ electronic transition in the dihydropyridine ring, while the hyperchromic effect in the $259-320 \mathrm{~nm}$ range is ascribed to 


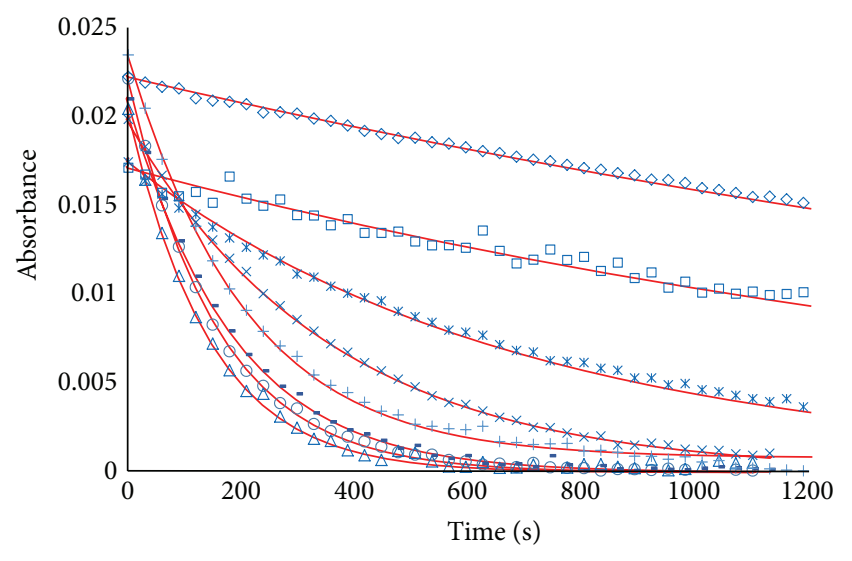

Irradiation wavelength $(\mathrm{nm})$

$\begin{array}{ll}\bigcirc 390 & \times 320 \\ \triangle 380 & * 281 \\ -370 & \diamond 259 \\ +345 & \square 235\end{array}$

FIGURE 2: The photokinetic traces of NIS in ethanol $\left(7.84 \times 10^{-6} \mathrm{M}\right)$ at $\lambda_{\text {irr }}=235,259,281,320,345,370,380$, and $390 \mathrm{~nm}$ and observed at $\lambda_{\text {obs }}=390 \mathrm{~nm}$. The symbols represent the experimental data while the lines represent the model fitted traces using the appropriate model equation.

the $\pi \rightarrow \pi^{*}$ electronic transitions in the aromatic ring [11]. The latter may also involve an underlying $\pi \rightarrow \pi^{*}$ transition of the dihydropyridine in agreement with the spectral contribution of each chromophore to the overall NIS electronic spectrum [17].

The primary photoprocess of NIS photodegradation is the occurrence of an intramolecular electron transfer from the dihydropyridine to the nitrophenyl group leading to a radical ion pair $[14,18]$. The favourable pathway for the decay of the latter intermediate involves a proton transfer followed by the elimination of a water molecule to produce the primary photoproduct (PP, Scheme 1) $[14,19]$. The spatial proximity of the oxygen atom of the ortho-nitro group on the phenyl ring to the hydrogen atom of the noncoplanar dihydropyridine moiety favours the proton transfer which represents a key step in the phototransformation of NIS $[14,19,20]$.

4.2. Modelling NIS Photodegradation Kinetics. NIS photodegradation kinetics was studied by exposing freshly prepared ethanolic solutions to different irradiation wavelengths $\left(\lambda_{\text {irr }}=259,281,320,345,370,380\right.$, and $\left.390 \mathrm{~nm}\right)$ spanning different regions of its absorption spectrum including its isosbestic points (259 and $320 \mathrm{~nm}$ ).

The variation of absorbance over time for each irradiation wavelength was recorded at a unique observation wavelength $\left(\lambda_{\text {obs }}=390 \mathrm{~nm}\right)$. These traces were labelled by their irradiation/observation wavelengths $\left(\lambda_{\text {irr }} / \lambda_{\text {obs }}\right)$. Subsequently, the fitting of the experimental traces was conducted using the suitable model equation (i.e., (1) or (5) for nonisosbestic and isosbestic irradiations, resp.) (Figure 2).

All the experimental traces were well fitted by the appropriate equation, thus confirming that (i) the mechanism of
NIS involves a unimolecular photoreaction as set out in Scheme 1, (ii) NIS photodegradation obeys a $\Phi$-order kinetics, and (iii) the model equation (1) is valid in describing $\mathrm{AB}(1 \Phi)$ photoreactions kinetics at any nonisosbestic and monochromatic irradiation. Furthermore, a faster degradation was observed upon exposure to irradiation wavelengths in the 340-390 $\mathrm{nm}$ region compared to irradiations in the 235-320 nm region of the spectrum. This might suggest that NIS photodegradation causative spectral range spans the UVA/visible regions. However, since various experimental, spectroscopic, and reactants' parameters are involved in the equation of NIS photoreaction rate-constant (2), the former conclusion must be considered with caution.

4.3. Wavelength Effects on NIS Quantum Yield. Fitting the models to the kinetic traces allows the determination of the respective value of $k_{\text {NIS }}^{\lambda_{\text {irr }}}$, which together with its formula (2) or (6) and data from the experimental kinetic traces (Figure 2), allows to readily determine the quantum yield values of NIS photodegradation at any monochromatic irradiation (Table 1).

The quantum yield of NIS photodegradation increases by more than 85 times with increasing wavelength from 235 to $390 \mathrm{~nm}$ (Table 1). Its values are more significant $(>0.1)$ for wavelengths higher than $280 \mathrm{~nm}$. It is, however, clear that the electronic transition situated in the $320-390 \mathrm{~nm}$ region is responsible for the highest phototransformation of NIS $\left(\Phi_{\text {NIS } \rightarrow \text { PP }}^{\lambda_{\text {irr }}}>0.3\right)$. The present values of $\Phi_{\text {NIS } \rightarrow \text { PP }}^{\lambda_{\text {irr }}}$ may suggest that the lowest singlet-state of the molecule is much more efficient in the photoconversion of NIS than are the other higher excited states, which underlines the important contribution of the $\pi \rightarrow \pi^{*}$ electronic transition of the dihydropyridine ring in the photochemistry of NIS.

Our $\Phi_{\text {NIS } \rightarrow \text { PP }}^{\lambda_{\text {irr }}}$ values agree with those reported in the literature for NIS in methanol as 0.35 at $\lambda_{\text {irr }}=366 \mathrm{~nm}$ [14] and $\lambda_{\text {irr }}=365 \mathrm{~nm}$ [11]. However, our $\Phi_{\mathrm{NIS} \rightarrow \mathrm{PP}}^{259}$ is more than 10 -fold lower than the reported value $\left(\Phi_{\mathrm{NIS} \rightarrow \mathrm{PP}}^{\lambda_{\text {ir }}}=0.25\right)$ for $\lambda_{\text {irr }}=254 \mathrm{~nm}$ [14]. This discrepancy might be due to the large bandwidth of the filtered irradiation beam (i.e., a polychromatic light) used for the latter study which can only lead to average quantum yield values. The effect of the polychromatic irradiation beam which is clearly much more pronounced at $254 \mathrm{~nm}$ than at $366 \mathrm{~nm}$ might be explained by a steeper variation of NIS quantum yield values in the 250$300 \mathrm{~nm}$ than in the 350-390 $\mathrm{nm}$ region (Table 1 and Figure 3 ). However, the $\Phi_{\text {NIS } \rightarrow \text { PP }}^{\lambda_{\text {irr }}}$ obtained in the present study match well the overall wavelength variation of the quantum yields observed for a NIS analogue, nifedipine [3]. Incidentally, the similarity between NIS and nifedipine results indicate that the extension of the alkyl chain (R) on the ester (-COO-R) group (a methyl in nifedipine) does not significantly affect the photochemical efficiency of the main structural dihydropyridinephenyl backbone. Also, the presence of the nitro group at the ortho-position of the aromatic ring in some dihydropyridine members such as NIS and nifedipine renders such molecules more prone to photolability as suggested by the relatively high quantum yields recorded for both molecules compared to those reported for other dihydropyridine members [21]. 
TABLE 1: Quantum yields and reaction parameter values for the photodegradation of NIS under different monochromatic irradiation wavelengths.

\begin{tabular}{lcccc}
\hline$\lambda_{\text {irr }} / \mathrm{nm}$ & $A_{\mathrm{NIS}}^{\lambda \text { irr } / 390}(0)$ & $P_{\lambda \text { irr }} /$ einstein $\cdot \mathrm{s}^{-1} \mathrm{dm}^{-3}$ & $k_{\mathrm{NIS}}^{\lambda_{\text {irr }}} \times 10^{3} / \mathrm{s}^{-1}$ & $\Phi_{\mathrm{NIS} \rightarrow \mathrm{PP}}^{\lambda_{\text {irr }}}$ \\
\hline 235 & 0.0171 & $8.64 \times 10^{-7}$ & 0.500 & $0.0041 \pm 0.0001$ \\
259 & 0.0222 & $2.64 \times 10^{-7}$ & 0.335 & $0.028 \pm 0.0111$ \\
281 & 0.0174 & $4.98 \times 10^{-7}$ & 1.240 & $0.122 \pm 0.0085$ \\
320 & 0.0198 & $3.53 \times 10^{-7}$ & 2.800 & $0.304 \pm 0.0157$ \\
345 & 0.0235 & $5.99 \times 10^{-7}$ & 5.100 & $0.326 \pm 0.0088$ \\
370 & 0.0210 & $6.32 \times 10^{-7}$ & 5.700 & $0.334 \pm 0.0200$ \\
380 & 0.0204 & $8.04 \times 10^{-7}$ & 7.300 & $0.345 \pm 0.0179$ \\
390 & 0.0221 & $1.02 \times 10^{-6}$ & 6.500 & $0.351 \pm 0.0069$ \\
\hline
\end{tabular}

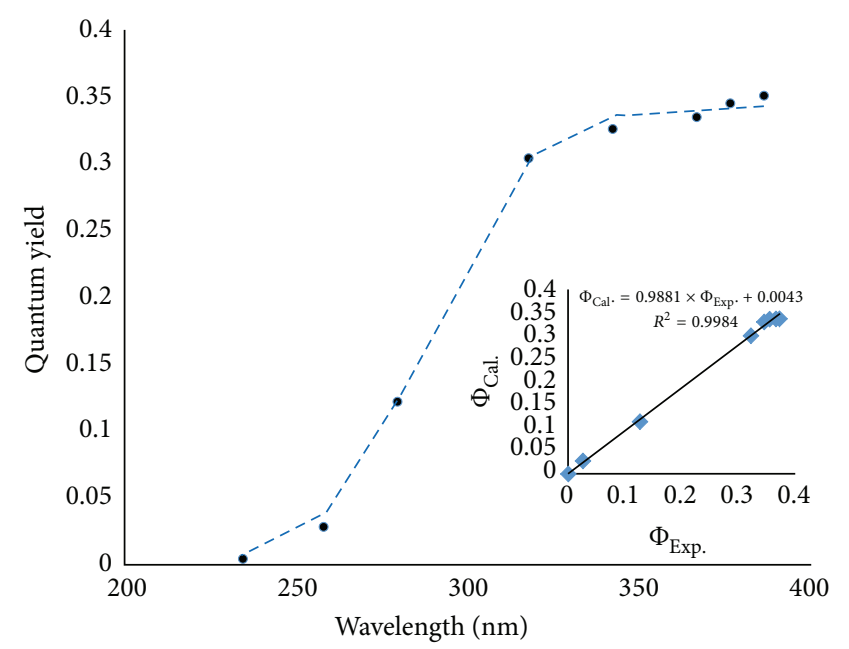

FIGURE 3: Sigmoid-like pattern of NIS photodegradation quantum yield values with irradiation wavelength. Inset: experimental $\left(\Phi_{\text {Exp. }}^{\lambda_{\text {irr }}}\right)$ versus calculated $\left(\Phi_{\text {Cal. }}^{\lambda_{\text {irr }}}\right)$ quantum yield values.

In any case, $\Phi_{\text {NIS } \rightarrow \text { PP }}^{\lambda_{\text {irr }}}$ values indicate that the UVA and the earliest section of the visible region are most efficient in NIS photodegradation. From a more general view point, our findings on the quantum yield dependence on irradiation wavelength as it has been observed for NIS, nifedipine [3], montelukast [5], fluvoxamine [6], and sunitinib [7] strongly underline the necessity of using monochromatic light for the determination of reliable values of quantum yields.

Besides, our NIS photodegradation quantum yields are well correlated with the irradiation wavelength (Figure 3) via a well-defined sigmoidal pattern (7). A good agreement is found between experimental and calculated (7) values of the quantum yields (as indicated by the linear relationship in the inset of Figure 3 ). Therefore, the sigmoid equation may be found useful to estimate the quantum yield absolute values at any irradiation wavelength between 235 and $390 \mathrm{~nm}$ :

$$
\Phi_{\mathrm{NIS} \rightarrow \mathrm{PP}}^{\lambda_{\mathrm{irr}}}=\frac{0.26}{0.76+50 \times e^{-0.07\left(\lambda_{\mathrm{irr}}-230\right)}} .
$$

4.4. NIS Initial Concentration Effect on Its Photodegradation. Various concentrations of NIS in ethanol were selected within NIS linearity range $\left(3.92 \times 10^{-6} \mathrm{M}\right.$ to $\left.1.58 \times 10^{-4} \mathrm{M}\right)$ to

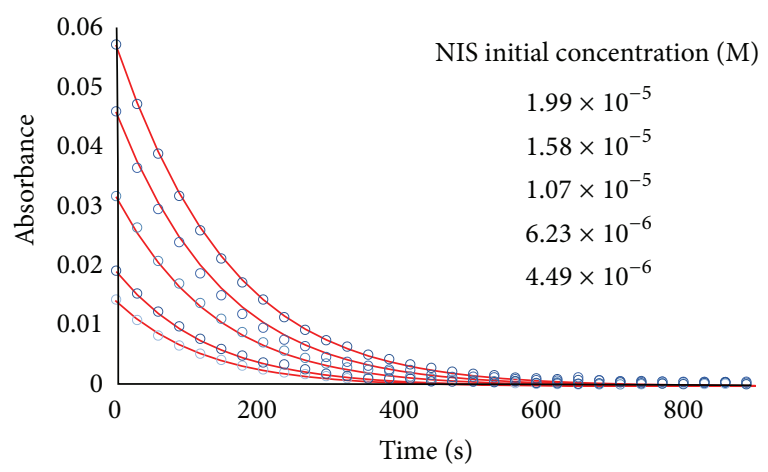

FIGURE 4: Photokinetic traces of NIS ethanolic solutions of various concentrations $\left(4.49 \times 10^{-6}-1.99 \times 10^{-5}\right)$ when irradiated at $350 \mathrm{~nm}$ and observed at $390 \mathrm{~nm}$.

investigate their effect on NIS photodegradation rate. This was performed by subjecting each freshly prepared solution to a particular irradiation wavelengths $\left(\lambda_{\text {irr }}=320,350\right.$, or $390 \mathrm{~nm}$ ), monitoring the absorbance at $\lambda_{\text {obs }}=390 \mathrm{~nm}$ and maintaining the radiant power unchanged for the whole set of experiments realised for any given $\lambda_{\text {irr }}$.

The kinetic profiles and the determined overall rateconstants are presented in Table 2 and Figure 4. The model equation fits well the traces with a $20 \%$ decrease of the $k_{\text {NIS }}^{\lambda_{\text {irr }}}$ values observed for a 5-fold increase in initial concentration for irradiation 320 and $350 \mathrm{~nm}$ whereas less than $6 \%$ variation was recorded for an 8-fold increase of NIS initial concentration at $\lambda_{\text {irr }}=390 \mathrm{~nm}$.

The conditions imposed on the present experiments (as set out above and more specifically for nonisosbestic irradiations) meant that only the photokinetic factor's value within the rate-constant equation is affected by a change in the initial concentration (as all the other variables were kept unchanged between different experiments). As expected from the formulae of $k_{\text {NIS }}^{\lambda_{\text {irr }}}$ (2) and $F_{\text {PP }}^{\lambda_{\text {irr }}}$ (3), the higher the NIS initial concentration the lower the $k_{\text {NIS }}^{\lambda_{\text {irr }}}$ value. Indeed, a linear relationship is established between these two factors for NIS for all nonisosbestic irradiations (320 and $350 \mathrm{~nm}$ ) and/or initial concentration sets studied (Figure 5). The slight variation of $k_{\text {NIS }}^{\lambda_{\text {irr }}}$ with NIS initial concentration when the irradiation is performed at $390 \mathrm{~nm}$ is also coherent with (2) 


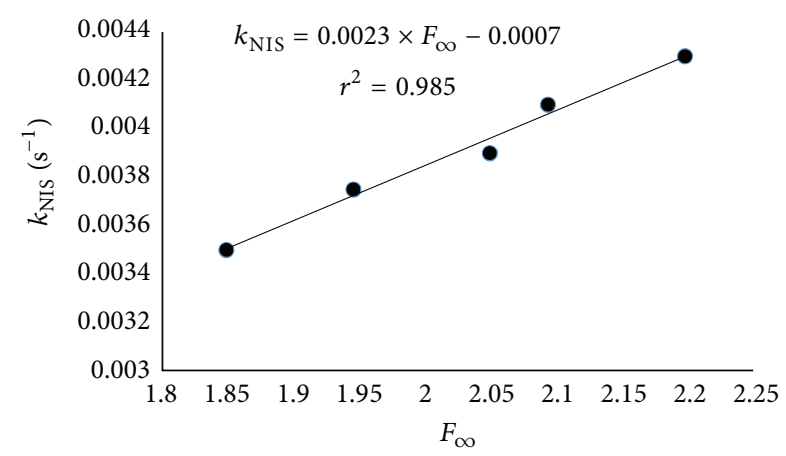

FIGURE 5: A linear relationship between the overall rate-constant of NIS photodegradation (obtained as the fitting parameter of the kinetic data with (1)) and the photokinetic factor of the photoproduct (calculated using (3)) when irradiating various concentrations of NIS solutions $\left(0.39-2.06 \times 10^{-5} \mathrm{M}\right)$ at $320 \mathrm{~nm}$ and observing at $390 \mathrm{~nm}$.

TABLE 2: Initial concentrations and overall rate-constants of NIS photodegradation measured at various irradiation wavelengths.

\begin{tabular}{cccc}
\hline$\lambda_{\text {irr }}$ & $A_{\mathrm{NIS}}^{\lambda_{\text {irr }} / 390}(0)$ & $k_{\mathrm{NIS}}^{\lambda_{\text {irr }}} / \mathrm{s}^{-1}$ & $C_{\mathrm{NIS}}(0) \times 10^{5} / \mathrm{M}$ \\
\hline \multirow{3}{*}{320} & 0.013 & 0.0043 & 0.396 \\
& 0.025 & 0.0041 & 0.826 \\
& 0.03 & 0.0039 & 1.01 \\
& 0.045 & 0.00375 & 1.53 \\
350 & 0.059 & 0.0035 & 2.06 \\
\hline \multirow{3}{*}{390} & 0.014 & 0.0082 & 0.449 \\
& 0.019 & 0.0080 & 0.623 \\
& 0.032 & 0.0077 & 1.07 \\
& 0.046 & 0.0075 & 1.58 \\
& 0.057 & 0.0072 & 1.99 \\
\hline \multirow{3}{*}{35} & 0.011 & 0.007 & 0.35 \\
& 0.022 & 0.007 & 0.73 \\
& 0.033 & 0.0069 & 1.12 \\
& 0.054 & 0.0069 & 1.86 \\
\hline
\end{tabular}

as the value of $F_{\infty}^{\lambda_{\text {irr }}}$ at the irradiation wavelength $(390 \mathrm{~nm}$, where only NIS absorbs) is constant for all the concentrations $\left(F_{\mathrm{O}}^{\lambda_{\text {irr }}}=F_{\mathrm{PP}}^{\lambda_{\text {irr }}} \approx 2.3\right)$.

The self-stabilization effect induced by the drug's initial concentration value, which works here for both isosbestic and nonisosbestic monochromatic-irradiation, would also be expected to hold for irradiation with polychromatic light and can be postulated for drug mechanisms that involve more than a single unimolecular photoreaction, $\mathrm{AB}(1 \Phi)$ (e.g., it has been proven for photoreversible reactions at any irradiation wavelength [4], and for cyclic trimolecular systems involving up to six photochemical steps when subjected to isosbestic irradiation [16].

The concept of photostabilisation through incrementation of the initial drug concentration could in fact explain a number of experimental observations reported in the literature for many drugs $[22,23]$. For instance, the postulated change of reaction-order for NIS photodegradation kinetics, which was attributed an apparent first-order kinetics for initial concentrations up to $3 \times 10^{-5} \mathrm{M}$ and a zero-order kinetics for concentrations above $2 \times 10^{-4} \mathrm{M}$ [12], might be interpreted as a photoreaction slow down due to increasing initial concentration. From the correlation line in Figure 5, a 2.75 -fold reduction in $k_{\text {NIS }}^{\lambda_{\text {irr }}}$ can be estimated to occur for the above concentrations. This would imply that the kinetic trace would have been steeper for lower initial NIS concentrations than higher ones and, therefore, might have justified the fitting of the former with the exponential model of the first-order reaction and the latter with the linear zeroorder reaction model. A similar misinterpretation can, for instance, be made for NIS kinetic trace at $235 \mathrm{~nm}$ irradiation (Figure 2), which could be fitted with zero-order kinetics while the much steeper trace resulting from irradiation at $390 \mathrm{~nm}$ (Figure 2) could be fitted with the first-order kinetics. This reiterates the need for an adequate kinetic method for photodegradation reactions since obtaining a good fitting with classical treatments evidently cannot be informative enough to reach reasonable conclusions on the relative reactivity of photodrugs.

A significant photostabilisation of NIS (and probably of many other drugs) can arguably be achieved by increasing its concentration, although this approach might considerably be limited by therapeutic dosages.

4.5. Quantification of NIS Photostabilisation with ExcipientDyes. Light absorption competitors have been proposed as a means to photostabilise photolabile drugs [22-26]. Redcoloured iron oxides are employed in many solid (tablet) formulations for this purpose [22, 27]. In terms of interpretation, the reaction slowdown observed when increasing the concentrations of indigotin and azorubine, for instance, was construed as resulting from a decrease in NIS photodegradation quantum yield in methanolic solutions. The dyes were believed to act as quenchers of nisoldipine excited tripletstate as Stern-Volmer constructs had confirmed [11]. The photodegradation rate-constant of nisoldipine in ethanol was also shown to decrease with increasing concentrations of $\beta$ carotene [15]. These studies based their treatments on firstorder kinetics and their conclusions were mainly reached by comparing the reactions rate-constant values. However, a comprehensive quantification of the effects of such light absorption competitors on the photoreaction overall rateconstant has not been, thus far, rationalised by a physicochemical/mathematical treatment. In our previous study [3], the reduction of the rate-constant could be directly linked to increasing concentrations of the absorption-competitor using $\Phi$-order kinetic equations. In the present study, the additives tartrazine (TRZ) and quinoline yellow (QY) have been studied as examples of excipient-dyes capable of retarding NIS photodegradation in ethanol.

NIS kinetic traces obtained in the presence of increasing concentrations of excipient-dyes (TRZ and QY) were fitted with high accuracy to (1) (Figure 6). Increasing the dyes concentrations was found to be inversely proportional to the overall photodegradation rate-constant (Table 3 ). 
TABLE 3: Dyes absorbance, overall reaction rate-constants, photokinetic factors, and percentage reduction in reaction rates of NIS photodegradation in the presence of various concentrations of TRZ and QY when irradiated and observed at $390 \mathrm{~nm}$.

\begin{tabular}{|c|c|c|c|c|c|}
\hline & $A_{\mathrm{dye}}^{\lambda_{\mathrm{irr}}^{\mathrm{a}}}$ & $F_{\infty}^{\lambda_{\text {irr }}}$ & $k_{\mathrm{NIS}}^{\lambda_{\mathrm{irr}}} / \mathrm{s}^{-1}$ & $k_{\mathrm{NIS}}^{\lambda_{\mathrm{irr}}}\left(A_{\mathrm{dye}}^{\lambda_{\mathrm{irr}}}=0\right) / k_{\mathrm{NIS}}^{\lambda_{\mathrm{irr}}}\left(A_{\mathrm{dye}}^{\lambda_{\text {irr }}} \neq 0\right)$ & $\%$ reduction $^{\mathrm{b}}$ \\
\hline NIS $^{\mathrm{c}}$ & 0 & 2.30 & 0.0080 & 1 & 0 \\
\hline \multirow{4}{*}{ Tartrazine (TRZ) } & 0.379 & 1.54 & 0.0052 & 1.54 & 35 \\
\hline & 0.762 & 1.08 & 0.0039 & 2.05 & 51.25 \\
\hline & 1.147 & 0.81 & 0.0032 & 2.50 & 60.00 \\
\hline & 1.415 & 0.68 & 0.0022 & 3.64 & 72.5 \\
\hline \multirow{4}{*}{ Quinoline Yellow (QY) } & 0.261 & 1.73 & 0.0067 & 1.19 & 16.25 \\
\hline & 0.472 & 1.40 & 0.0055 & 1.45 & 31.25 \\
\hline & 0.792 & 1.06 & 0.0040 & 2.00 & 50 \\
\hline & 1.137 & 0.82 & 0.0032 & 2.50 & 60 \\
\hline
\end{tabular}

${ }^{a}$ Absorbance of the dye measured at the irradiation wavelength of $390 \mathrm{~nm}$ for concentrations given in Figure 7 .

${ }^{\mathrm{b}}$ The constant concentration of NIS was $1.56 \times 10^{-5} \mathrm{M}$.

${ }^{c}$ The radiant power value for the experiments was $P_{390}=1.74 \times 10^{-6}-1.78 \times 10^{-6}$ einstein $\cdot \mathrm{dm}^{-3} \cdot \mathrm{s}^{-1}$.

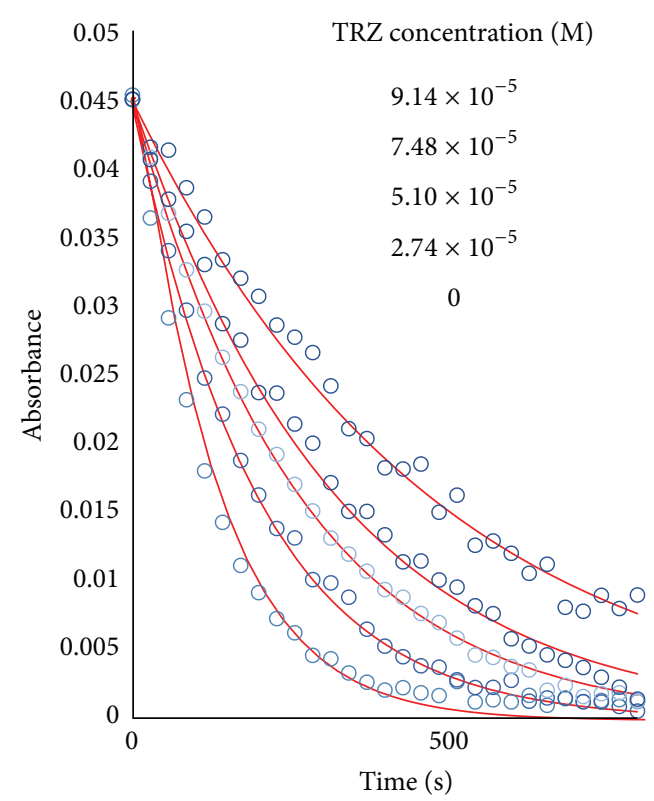

(a)

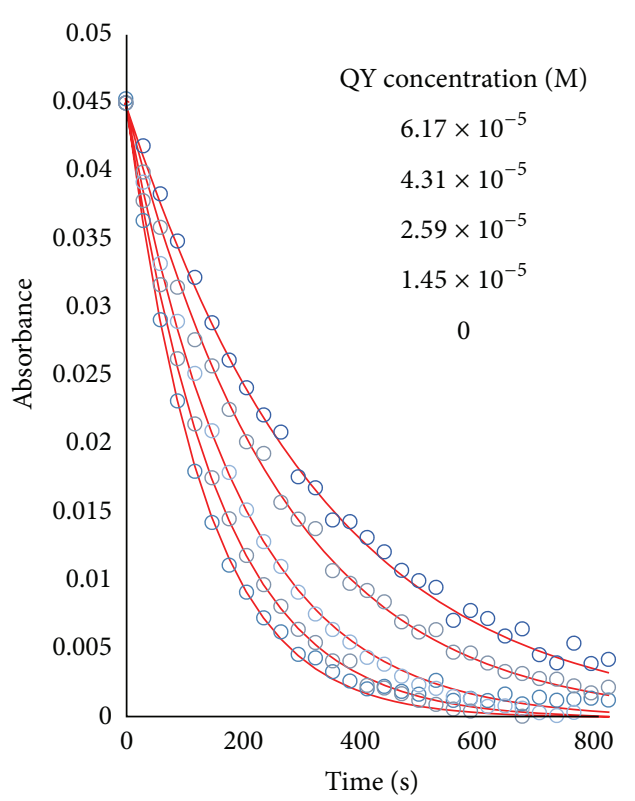

(b)

FIGURE 6: Effect of increasing (a) TRZ and (b) QY concentrations on the photodegradation traces of $1.56 \times 10^{-5} \mathrm{M}$ NIS solutions when irradiated and observed at $390 \mathrm{~nm}$.

A good linear correlation is found between the values of the photokinetic factor $\left(F_{\mathrm{o}}^{\lambda_{\text {irr }}}\right)$ and the overall rate-constant $\left(k_{\mathrm{NIS}}^{\lambda_{\mathrm{irr}}}\right)$ when the former is calculated by involving the values of the absorbances of the dyes in the expression of the medium's total absorbance as $A_{\text {tot }}^{\lambda_{\text {irr }} / \lambda_{\text {irr }}}(\infty)=A_{\mathrm{PP}}^{\lambda_{\text {irr }} / \lambda_{\text {irr }}}(\infty)+A_{\text {Excip. }}^{\lambda_{\text {irr }} / \lambda_{\text {irr }}}($ Figure 7, with $\lambda_{\text {irr }}=390 \mathrm{~nm}$ and $A_{\mathrm{PP}}^{390 / 390}(\infty)=0$ ). As a consequence, the higher the dye's absorbance, the smaller the $F_{\infty}^{\lambda_{\text {irr }}}$ value that leads to significant reduction of $k_{\mathrm{NIS}}^{\lambda_{\text {irr }}}$ values (Figure 7, Table 3). Therefore, the true action of the light competitor on the photodegradation reaction is revealed in the reduction of the photokinetic factor value for increasing excipient-dye concentrations but does not affect NIS quantum yield as might have been suggested [11].

4.6. NIS Actinometric Potential. The ICH recommended quinine hydrochloride actinometer for photostability testing purposes [8] has been found to suffer from a number of limitations including but not limited to an ill-defined photochemical mechanism and a persistent thermal degradation in the dark [28]. These drawbacks seriously hamper the reliability of this actinometer. It is also noticeable that quinine hydrochloride has rarely been employed for the investigations of drugs' photodegradation in solution $[22,29,30]$. 


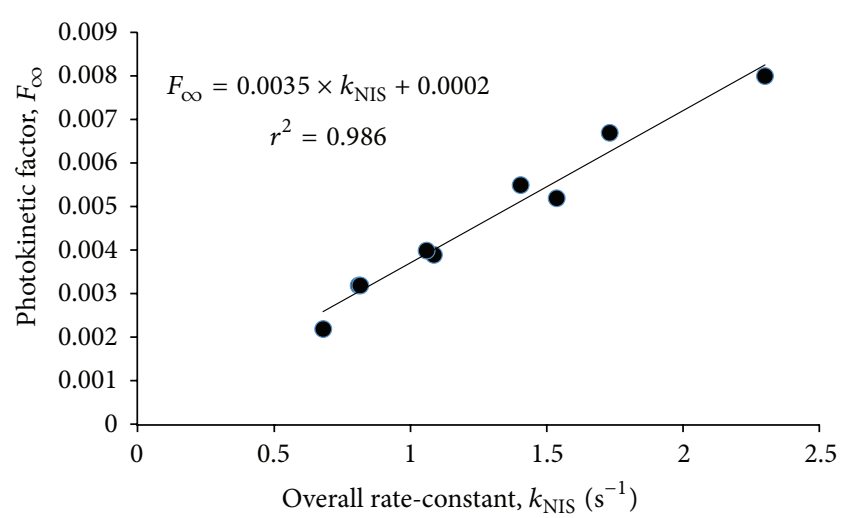

FIGURE 7: Linear relationship between $F_{\infty}^{\lambda_{\text {irr }}}$ and $k_{\text {NIS }}^{\lambda_{\text {irr }}}$ for data collected for both TRZ and QY various concentrations (Table 3 ) in the presence of $1.56 \times 10^{-5}$ M NIS irradiated and observed at $390 \mathrm{~nm}$.

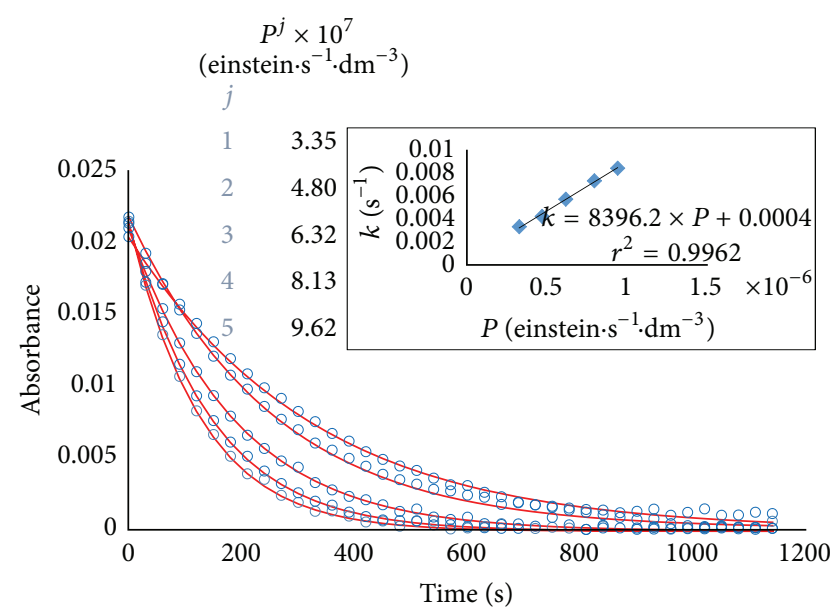

FIGURE 8: Effect of increasing radiant power, $P_{370}^{j}$, on NIS kinetic traces 370/390. The circles represent the experimental traces and the lines represent the fitting traces using (1). Inset: correlation between $k_{\mathrm{NIS}, j}^{\lambda_{\text {irr }, i}}$ of each kinetic trace and its corresponding $P_{370}^{j}$.

Nevertheless, no alternative actinometers have thus far been proposed. In this respect, the equations for $\Phi$-order kinetics have enabled the development of new $\mathrm{AB}(1 \Phi)$ actinometers including conventional drug molecules [2, 3, 5-7].

Accordingly, the usefulness of NIS for actinometry was established by selecting four $(i=4)$ irradiation wavelengths $(320,345,370$, and $390 \mathrm{~nm})$ within the region of fast photodegradation. For each wavelength, a set of “ $j$ ” different radiant power values (spanning our instrumentation capacities) were performed on separate NIS solutions of the same concentration. The resulting traces were well fitted by (1) (Figure 8), which then allowed the values of $k_{\mathrm{NIS}, j}^{\lambda_{\text {irr, }} \text { to be }}$ determined for each trace $\left(\lambda_{\mathrm{irr}, i}, P_{\lambda_{\mathrm{irr}, i}}^{j}\right)$.

Increasing the radiant power at any irradiation wavelength had the effect of accelerating the rate of the photoreaction. As predicted by (2), good linear correlations were found between $k_{\mathrm{NIS}, j}^{\lambda_{\text {irr, }}}$ and the corresponding $P_{\lambda_{\text {irr. } i}^{j}}^{j}$ values

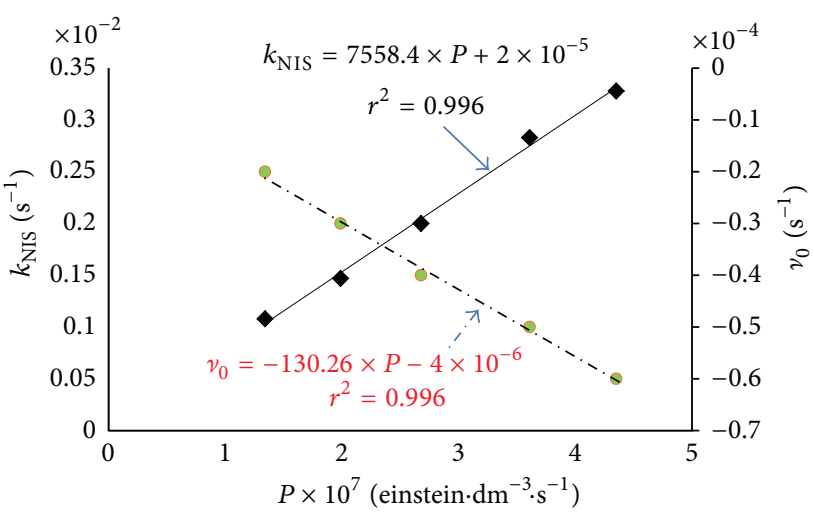

Figure 9: Linear correlations between the parameters of the traces $k_{\mathrm{NIS}, j}^{320}$ and $\nu_{0, j}^{320 / 390}$ against the radiant power, $P_{320}^{j}$ for NIS in ethanol. The irradiation/observation wavelengths were 320/390, and the $j$ values of the radiant power were taken from Table 4.

(Figure 9 and Table 4), with intercepts approaching a zero value and correlation coefficients of almost unity. The gradient of the equation was expressed as a factor $\beta_{\lambda_{\text {irr }}}(2)$.

In general, such linear relationships are expected to be found irrespective of NIS initial concentration (as long as $\left.A_{\mathrm{PP}}^{\lambda_{\text {irr }} / \lambda_{\text {irr }}}(\infty)<0.65\right)$, and/or the radiant power value (that can be a millionfold higher than $P_{\lambda_{\text {irri }}^{j}}^{j}$ used in this study [3]).

It is also interesting to notice that from an application viewpoint, the variation of $k_{\mathrm{NIS}, j}^{\lambda_{\text {irr }, i}}$ with $P_{\lambda_{\text {irr.i }}^{j}}^{j}$ can be investigated independently of and prior to knowing the quantum yield of the $\mathrm{AB}(1 \Phi)$ system at hand. This represents an advantage in developing new drug-actinometers.

Furthermore, the actinometric study can be even simpler to carry out if instead of using $k_{\mathrm{NIS}, j}^{\lambda_{\mathrm{irr}, i}}$ as a dependent variable, the initial reaction-rate $\left(\nu_{0, j}^{\lambda_{\text {irr, }} / \lambda_{\text {obs }}},(4)\right)$ is employed. Indeed, the values of $\nu_{0, j}^{\lambda_{\text {irr, } i} / \lambda_{\text {obs }}}$ for the $j$ radiant power experiments for the $i$ th irradiation wavelength can readily be obtained as the gradients $\left(\delta_{\lambda_{\text {irr }}}\right)$ of the linear relationships fitting the very first experimental points of each photodegradation trace (Table 4, Figure 8). Obviously, the determination of the $\delta_{\lambda_{\text {irr }}}$ values for $\mathrm{AB}(1 \Phi)$ systems does not require the prior knowledge of both reaction attributes' and experimental parameters' values and/or a complete trace. It is worth noting that $\delta_{\lambda_{\text {irr }}}$ and $\beta_{\lambda_{\text {irr }}}$ factors express different quantities as given by (2) and (4) and hence do not directly correlate with each other.

The values of the gradients $\left(\beta_{\lambda_{\text {irr }}}\right.$ or $\left.\delta_{\lambda_{\text {irr }}}\right)$ offer the possibility of determining the radiant power value of any monochromatic irradiation beam between 320 and $400 \mathrm{~nm}$, by proceeding as follows: (a) irradiate NIS (or any $\mathrm{AB}(1 \Phi)$ system) at the selected wavelength and subsequently (b) determine either its rate-constant (by fitting the semiempirical integrated rate-law model, (1)) or determining its initial rate of photoreaction. The corresponding value of $\beta_{\lambda_{\text {irr }}}$ (c) is worked out from one of the linear equations set out in Figure 10 and (d) the appropriate equation (for either $k_{\mathrm{NIS}}^{\lambda_{\text {irr }}}$ or $\nu_{0(i, j)}^{\lambda_{\text {irr }} / \lambda_{\text {obs }}}$ ) 
TABLE 4: Correlation equations for the variation of NIS photodegradation overall rate-constants $\left(k_{\mathrm{NIS}}^{\lambda_{\text {irr }}}\right)$ and initial reaction velocities $\left(\nu_{0}^{\lambda_{\text {irr }} / \lambda_{\text {obs }}}\right)$ with radiant power $\left(P_{\lambda_{\text {irr }}}\right)$, the corresponding $\beta_{\lambda_{\text {irr }}}$ and $\delta_{\lambda_{\text {irr }}}$ factors, and the span of radiant power employed for various monochromatic irradiations.

\begin{tabular}{|c|c|c|c|}
\hline $\begin{array}{l}\text { Irradiation } \\
\text { wavelength } \lambda_{\text {irr }} / \mathrm{nm}\end{array}$ & Equation of the line $\mathrm{a}^{\mathrm{a}}$ & Correlation coefficient, $r^{2}$ & $P_{\lambda_{\text {irr }}} \times 10^{7} /$ einst $\cdot \mathrm{s}^{-1} \cdot \mathrm{dm}^{-3}$ \\
\hline \multicolumn{4}{|c|}{$k_{\mathrm{NIS}}^{\lambda_{\mathrm{irr}}}=\beta_{\lambda_{\mathrm{irr}}} \times P_{\lambda_{\text {irr }}}+$ intercept } \\
\hline 390 & $7902.7 \times P_{390}-5 \times 10^{-6}$ & 0.996 & $3.81-10.9$ \\
\hline 370 & $8850.8 \times P_{370}+5 \times 10^{-7}$ & 0.999 & $5.55-11.9$ \\
\hline 345 & $8391.1 \times P_{345}-7 \times 10^{-6}$ & 0.998 & $2.42-7.20$ \\
\hline 320 & $7558.4 \times P_{320}+2 \times 10^{-5}$ & 0.996 & $1.34-4.35$ \\
\hline \multicolumn{4}{|c|}{$\nu_{0}^{\lambda_{\text {irr }} / \lambda_{\text {obs }}}=\delta_{\lambda_{\text {irr }}} \times P_{\lambda_{\text {irr }}}+$ intercept } \\
\hline 390 & $-97.98 \times P_{390}-5 \times 10^{-6}$ & 0.944 & $3.81-10.9$ \\
\hline 370 & $-226.05 \times P_{370}+7 \times 10^{-5}$ & 0.956 & $5.55-11.9$ \\
\hline 345 & $-124 \times P_{345}-1 \times 10^{-5}$ & 0.984 & $2.42-7.20$ \\
\hline 320 & $-130.26 \times P_{320}-4 \times 10^{-6}$ & 0.996 & $1.34-4.35$ \\
\hline
\end{tabular}

${ }^{\mathrm{a}} k_{\mathrm{NIS}}^{\lambda_{\text {irr }}}, \nu_{0}^{\lambda_{\text {irr }} / \lambda_{\text {obs }}}$, and intercepts expressed in $\mathrm{s}^{-1} ; \beta_{\lambda_{\text {irr }}}$ and $\delta_{\lambda_{\text {irr }}}$ in einst ${ }^{-1} \cdot \mathrm{dm}^{3}$.

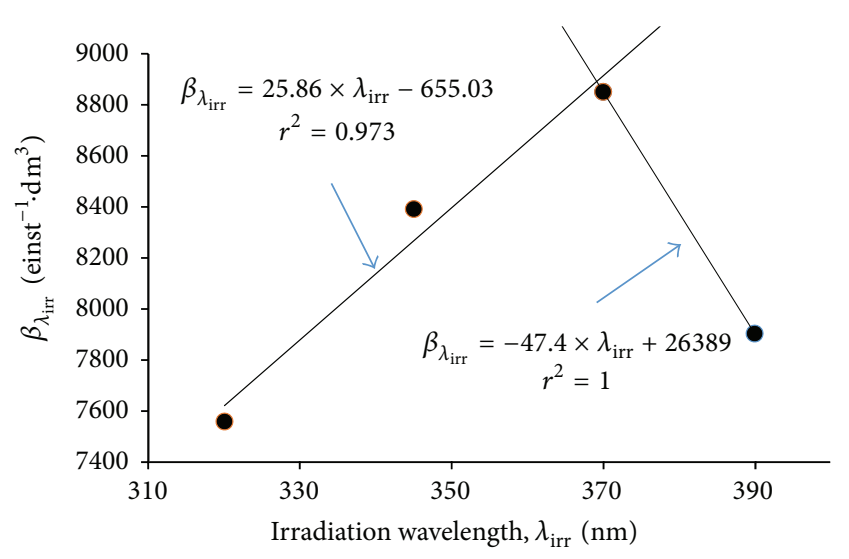

FIGURE 10: Variation of $\beta_{\lambda_{\text {irr }}}$ factors with irradiation wavelengths (data from Table 4).

(8) can then be used to determine the value of the radiant power $P_{\lambda_{\text {irr }}}$ of the light-source at the selected wavelength; the following was written by ignoring the lines' intercepts which have approximately zero values, Figure 9:

$$
P_{\lambda_{\text {irr }}}=\frac{k_{\mathrm{NIS}}^{\lambda_{\text {irr }}}}{\beta_{\lambda_{\text {irr }}}}=\frac{\nu_{0(i, j)}^{\lambda_{\text {irr }} / \lambda_{\text {obs }}}}{\delta_{\lambda_{\text {irr }}}} .
$$

Equation (8) should apply for any experiment if NIS initial concentration and the irradiation path-length are similar to the ones used in this study $\left(C_{\mathrm{NIS}}(0)=7 \times 10^{-6} \mathrm{M}\right.$ and $\left.l_{\text {irr }}=2 \mathrm{~cm}\right)$. However, if $C_{\mathrm{NIS}}(0)$ is the same but $l_{\text {irr,actual }}$ is different, then a small adjustment must first be made before the $\beta_{\lambda_{\text {irr }}}$ factor is used in (8). Mainly, the value of PP absorbance at the end of the reaction as measured for the actual reaction $\left(A_{\mathrm{PP}}^{\lambda_{\text {irr }} / \lambda_{\text {irr }}}(\infty)\right)$ will be used to calculate the photokinetic factor in two different ways, $F_{\infty \text {,actual }}^{\lambda_{\text {irr }}}$ using the optical path length $l_{\lambda_{\text {irractual }}}$ and the correcting $F_{\infty \text {,corr. }}^{\lambda_{\text {irr }}}$ using the optical path length of our experiment, $l_{\lambda_{\text {irr }}}=2 \mathrm{~cm}$. Then the $\beta_{\lambda_{\text {irr }}}$ factor (Figure 10) should be multiplied by the product $\left(F_{\infty \text {,actual }}^{\lambda_{\text {irr }}} \times l_{\lambda_{\text {irr,actual }}}\right)$ and divided by the product $\left(2 \times F_{\infty \text {,corr. }}^{\lambda_{\text {irr }}}\right)$.

Therefore, NIS could be used as an actinometer for monochromatic irradiations in the region of $320-400 \mathrm{~nm}$. Our method circumvents the problems encountered with many actinometers [31, 32] including the currently $\mathrm{ICH}$ recommended quinine actinometry [8], as the factors influencing the photoreaction of the actinometer (initial concentration, irradiation wavelength, absorption coefficient of the species, excitation, and monitoring optical path lengths) are all involved in (8). Overall, because our approach is easy-to-implement (especially through the treatment using the initial velocity), it may represent an interesting method for the development of additional new drug-actinometers. In fact, it has thus far allowed us to propose four new accurate actinometers covering the whole spectrum of light, namely, NIS (320-400 nm), nifedipine $(280-400 \mathrm{~nm})$ [3], montelukast $(258-380 \mathrm{~nm})$ [5], fluvoxamine $(260-290 \mathrm{~nm})$ [6], sunitinib (320-480) [7], and 1,2-bis[2-methylbenzo[b] thiophen-3,3,4,4,5,5-hexafluoro-1-cyclopentene, DAE, for the visible 405-570 $\mathrm{nm}$ region [2].

Furthermore, the above study is useful to shed light on some crucial aspects relating to photochemical reaction attributes. It is interesting to note that besides its utility in actinometry, $\beta_{\lambda_{\text {irr }}}$ also appears to be an important factor that reflects the rate of a particular photoreaction [6]. It can be compared between different studies using the same molecule at the same initial concentrations. This is because, $\beta_{\lambda_{\text {irr }}}$ does not depend on the radiant power which is hard to replicate between different experiments and especially when different research groups use different types of irradiation equipment. As such, unlike the overall rate-constant which is dependent on $P_{\lambda_{\text {irr }}}, \beta_{\lambda_{\text {irr }}}$ can be used to compare the rate of photodegradation of a particular molecule in different laboratory settings as long as the experiments are conducted using the same solvent, initial concentration, and irradiation wavelength (adjustment might be required when $l_{\lambda_{\text {irr }}} \neq 2$, as 
set out above). The $\beta_{\lambda_{\text {irr }}}$ factors might help solving the longstanding problem of comparing photodegradation results obtained in different conditions [22].

It becomes obvious from our results that the photodegradation causative range of the drug can neither be precisely defined on the basis of quantum yield nor on that of the overall rate-constant values. The former represents an absolute value of the efficiency of the photoreaction which does not depend on the reaction parameters such as the absorption coefficient of the photoproduct, whereas the latter depends on a combination of reactivity, reagents', and experimental parameters, whose values are mostly variable with wavelength; in addition to the fact that the magnitude of $P_{\lambda_{\text {irr }}}$ can also be arbitrarily set. Hence, for a given molecule the $\beta_{\lambda_{\text {irr }}}$ factor stands as a better criterion for the definition of the reaction performance than $\Phi_{\text {NIS } \rightarrow \text { PP }}^{\lambda_{\text {irr }}}$ or $k_{\text {NIS }}^{\lambda_{\text {irr }}}$, if the reactions performed to determine the $\beta_{\lambda_{\text {irr }}}$ factors were carried out with the same initial concentration of the drug. This stems from the fact that the $\beta_{\lambda_{\text {irr }}}$ factor, defined exclusively by the irradiation (not the observation) conditions, encompasses the contribution of the absorptivities of the reacting species, the efficiency of the photoreaction, the excitation optical path length, and the initial concentration, that is, all basic parameters influencing the rate of the photoreaction (with the exception of the radiant power). Therefore, the variation of the $\beta_{\lambda_{\text {irr }}}$ factors with wavelength can provide an effective tool to set the real limits for the photodegradation causative range of the drug (Figure 9).

For NIS, the $\beta_{\lambda_{\text {irr }}}$ factors variation with irradiation wavelength follows a triangular pattern (Figure 10). Accordingly, NIS photoreaction is fastest under 370-nm irradiation, and its photodegradation causative range is situated between 320 and $400 \mathrm{~nm}$ (considering here that $\beta_{\lambda_{\text {irr }}} \geq 7400$ einstein ${ }^{-1} . \mathrm{dm}^{3}$ ).

\section{Conclusion}

The recently developed $\mathrm{AB}(1 \Phi)$ model has successfully been applied to describe the kinetics of NIS photodegradation. This model can describe the kinetics of any $\mathrm{AB}(1 \Phi)$ system much more accurately than the classical treatments based on thermal reaction orders. NIS quantum yields of photodegradation were found to follow a sigmoid-like function with irradiation wavelength hence allowing the estimation of this parameter at any monochromatic irradiation wavelength in the $230-390 \mathrm{~nm}$ range. It has also become possible to quantify the self-stabilisation effect due to increasing the initial species concentration by a procedure using the rateconstant equation for $\mathrm{AB}(1 \Phi)$ systems. NIS photostabilisation by the presence of photostable excipient-dyes TRZ and QY, which act as absorption competitors as their electronic spectra overlap the same spectral range as NIS, has also been studied. Both species were shown to significantly reduce NIS photodegradation (up to $70 \%$, for concentrations within the linearity ranges of these dyes). The observed photostabilising effect has also been successfully rationalised using the simple $\mathrm{AB}(1 \Phi) \Phi$-order kinetics equations which are capable of describing and predicting the effect of any photoabsorbing photostable species at the selected irradiation wavelength. Finally, it was shown how NIS may be used as a simple and accurate actinometer for monochromatic irradiations within the 320-400 nm spectral range. A new approach of evaluating the photodegradation causative range is proposed on the basis of the variation of $\beta_{\lambda_{\text {irr }}}$ factor values with wavelength.

These findings confirm the usefulness and flexibility of the kinetic equations, set out for $\Phi$-order photoreactions of the $\mathrm{AB}(1 \Phi)$ type, to investigate a number of aspects relating to drug photodegradation, photostabilisation, and actinometry.

\section{Conflict of Interests}

The authors declare that there is no conflict of interests regarding the publication of this paper.

\section{References}

[1] M. Maafi and R. G. Brown, "The kinetic model for $\mathrm{AB}(1 \varphi)$ systems. A closed-form integration of the differential equation with a variable photokinetic factor," Journal of Photochemistry and Photobiology A: Chemistry, vol. 187, no. 2-3, pp. 319-324, 2007.

[2] M. Maafi, "The potential of $\mathrm{AB}(1 \Phi)$ systems for direct actinometry. Diarylethenes as successful actinometers for the visible range," Physical Chemistry Chemical Physics, vol. 12, no. 40, pp. 13248-13254, 2010.

[3] W. Maafi and M. Maafi, "Modelling nifedipine photodegradation, photostability and actinometric properties," International Journal of Pharmaceutics, vol. 456, no. 1, pp. 153-164, 2013.

[4] M. Maafi and W. Maafi, " $\Phi$-order kinetics of photoreversibledrug reactions," International Journal of Pharmaceutics, vol. 471, pp. 536-543, 2014.

[5] M. Maafi and W. Maafi, "Montelukast photodegradation: Elucidation of $\Phi$-order kinetics, determination of quantum yields and application to actinometry," International Journal of Pharmaceutics, vol. 471, pp. 544-552, 2014.

[6] M. Maafi and W. Maafi, "Quantitative assessment of photostability and photostabilisation of Fluvoxamine and its design for actinometry," Photochemical \& Photobiological Sciences, vol. 14, no. 5, pp. 982-994, 2015.

[7] M. Maafi and L. Y. Lee, "Actinometric and $\Phi$-order photodegradation properties of anti-cancer Sunitinib," Journal of Pharmaceutical and Biomedical Analysis, vol. 110, pp. 34-41, 2015.

[8] ICH, "Guidelines for industry Q1B photostability testing of new substances and products," Federal Register, vol. 62, pp. 2711527122, 1996.

[9] V. Marinkovic, D. Agbaba, K. Karljikovic-Rajic, J. Comor, and D. Zivanov-Stakic, "UV derivative spectrophotometric study of the photochemical degradation of nisoldipine," Il Farmaco, vol. 55, no. 2, pp. 128-133, 2000.

[10] A. Álvarez-Lueje, L. Naranjo, L. J. Núñez-Vergara, and J. A. Squella, "Electrochemical study of nisoldipine: analytical application in pharmaceutical forms and photodegradation," Journal of Pharmaceutical and Biomedical Analysis, vol. 16, no. 5, pp. 853-862, 1998.

[11] J. Mielcarek, W. Augustyniak, P. Grobelny, and G. Nowacka, "Photoprotection of 1,4-dihydropyridine derivatives by dyes," International Journal of Pharmaceutics, vol. 304, no. 1-2, pp. 145151, 2005.

[12] C. Huang, H. Liu, J. Ren, and X. Du, "Studies of photodegradation stability of nisoldipine," Chinese Journal of Pharmaceuticals, vol. 27, pp. 26-27, 1996. 
[13] V. D. Marinkovic, D. Agbaba, K. Karljikovic-Rajic, S. Vladimirov, and J. M. Nedeljkovic, "Photochemical degradation of solid-state nisoldipine monitored by HPLC," Journal of Pharmaceutical and Biomedical Analysis, vol. 32, no. 4-5, pp. 929-935, 2003.

[14] E. Fasani, D. Dondi, A. Ricci, and A. Albini, "Photochemistry of 4-(2-Nitrophenyl)-1,4-dihydropyridines. Evidence for electron transfer and formation of an intermediate," Photochemistry and Photobiology, vol. 82, no. 1, pp. 225-230, 2006.

[15] J. Mielcarek, P. Grobelny, and O. Szamburska, "The effect of $\beta$-carotene on the photostability of nisoldipine," Methods and Findings in Experimental and Clinical Pharmacology, vol. 27, no. 3, pp. 167-171, 2005.

[16] M. Maafi and R. G. Brown, "Photophysics and kinetics of naphthopyran derivatives. Part 1. General analytical solutions for the kinetics of $\mathrm{AB}(\mathrm{k}, \phi)$ and $\mathrm{ABC}(\mathrm{k}, \phi)$ systems," International Journal of Chemical Kinetics, vol. 37, no. 3, pp. 162-174, 2005.

[17] A. Krufurst and J. Kuhan, "Quantum chemical interpretation of electronic absorption spectra of the hantzsch dihydropyridines," Collection of Czechoslovak Chemical Communications, vol. 43, pp. 1422-1428, 1983.

[18] F. M. Martens, J. W. Verhoeven, C. A. G. O. Varma, and P. Bergwerf, "Photo-oxidation of 1,4-dihydropyridines by various electron acceptors: a laser flash photolysis study," Journal of Photochemistry, vol. 22, no. 2, pp. 99-113, 1983.

[19] X.-Q. Zhu, H.-R. Li, Q. Li et al., "Determination of the C4-H bond dissociation energies of NADH models and their radical cations in acetonitrile," Chemistry: A European Journal, vol. 9, no. 4, pp. 871-880, 2003.

[20] M. C. Ramusino and M. R. Varì, "Force field and semi-empirical MO conformational analysis of dihydropyridine calciumchannel antagonists," Journal of Molecular Structure: Theochem, vol. 492, pp. 257-268, 1999.

[21] E. Fasani, A. Albini, and M. Mella, "Photochemistry of Hantzsch 1,4-dihydropyridines and pyridines," Tetrahedron, vol. 64, no. 14, pp. 3190-3196, 2008.

[22] J. T. Piechocki and K. Thoma, Pharmaceutical Photostability and Photostabilisation Technology, Informa Healthcare, London, UK, 2010

[23] H. H. Tønnesen, Photostability of Drugs and Drug Formulations, CRC Press, London, UK, 2nd edition, 2004.

[24] R. C. Rowe, P. J. Sheskey, and P. J. Weller, Handbook of Pharmaceutical Excipients, Pharmaceutical Press and American Pharmaceutical Association, 2003.

[25] A. Albini and E. Fasani, Drugs Photochemistry and Photostability, The Royal Society of Chemistry, Cambridge, UK, 1998.

[26] K. Thoma and R. Klimek, "Photostabilization of drugs in dosage forms without protection from packaging materials," International Journal of Pharmaceutics, vol. 67, no. 2, pp. 169-175, 1991.

[27] D. S. Desai, M. A. Abdelnasser, B. A. Rubitski, and S. A. Varia, "Photostabilization of uncoated tablets of sorivudine and nifedipine by incorporation of synthetic iron oxides," International Journal of Pharmaceutics, vol. 103, no. 1, pp. 69-76, 1994.

[28] S. W. Baertschi, K. M. Alsante, and H. H. Tønnesen, "A critical assessment of the ICH guideline on photostability testing of new drug substances and products (Q1B): recommendation for revision," Journal of Pharmaceutical Sciences, vol. 99, no. 7, pp. 2934-2940, 2010.
[29] C. A. De Azevedo Filho, D. de Filgueiras Gomes, J. P. de Mélo Guedes, R. M. F. Batista, and B. S. Santos, "Considerations on the quinine actinometry calibration method used in photostability testing of pharmaceuticals," Journal of Pharmaceutical and Biomedical Analysis, vol. 54, no. 4, pp. 886-888, 2011.

[30] S. W. Baertschi, "Commentary on the quinine actinometry system described in the ICH draft guideline on photostability testing of new drug substances and products," Drug Stability, vol. 1, no. 4, pp. 193-195, 1997.

[31] H. J. Kuhn, S. E. Braslavsky, and R. Schmidt, "Chemical actinometry (IUPAC technical report)," Pure and Applied Chemistry, vol. 76, no. 12, pp. 2105-2146, 2004.

[32] M. Montalti, A. Credi, L. Prodi, and M. Teresa Gandolfi, Handbook of Photochemistry, CRC Press, Boca Raton, Fla, USA, 3rd edition, 2006. 

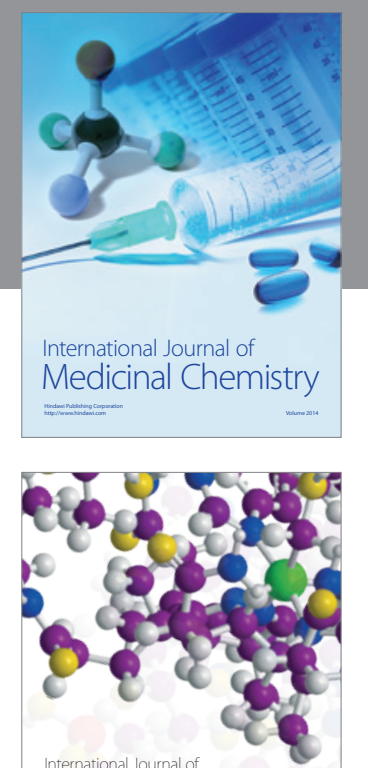

\section{Carbohydrate} Chemistry

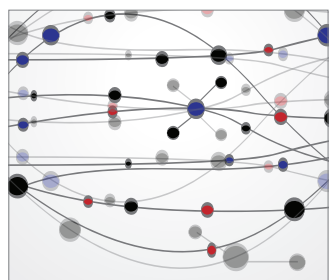

The Scientific World Journal
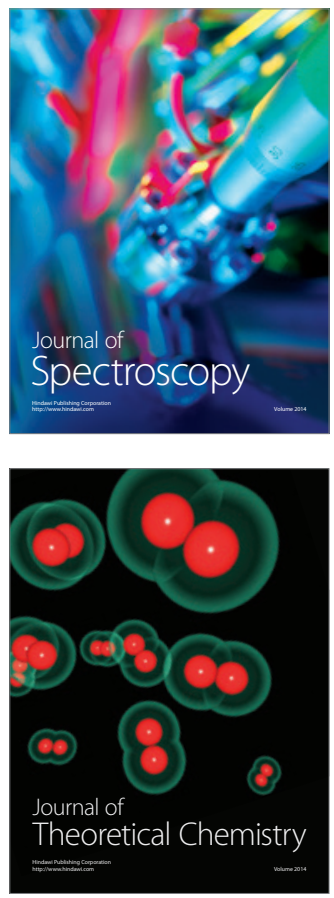
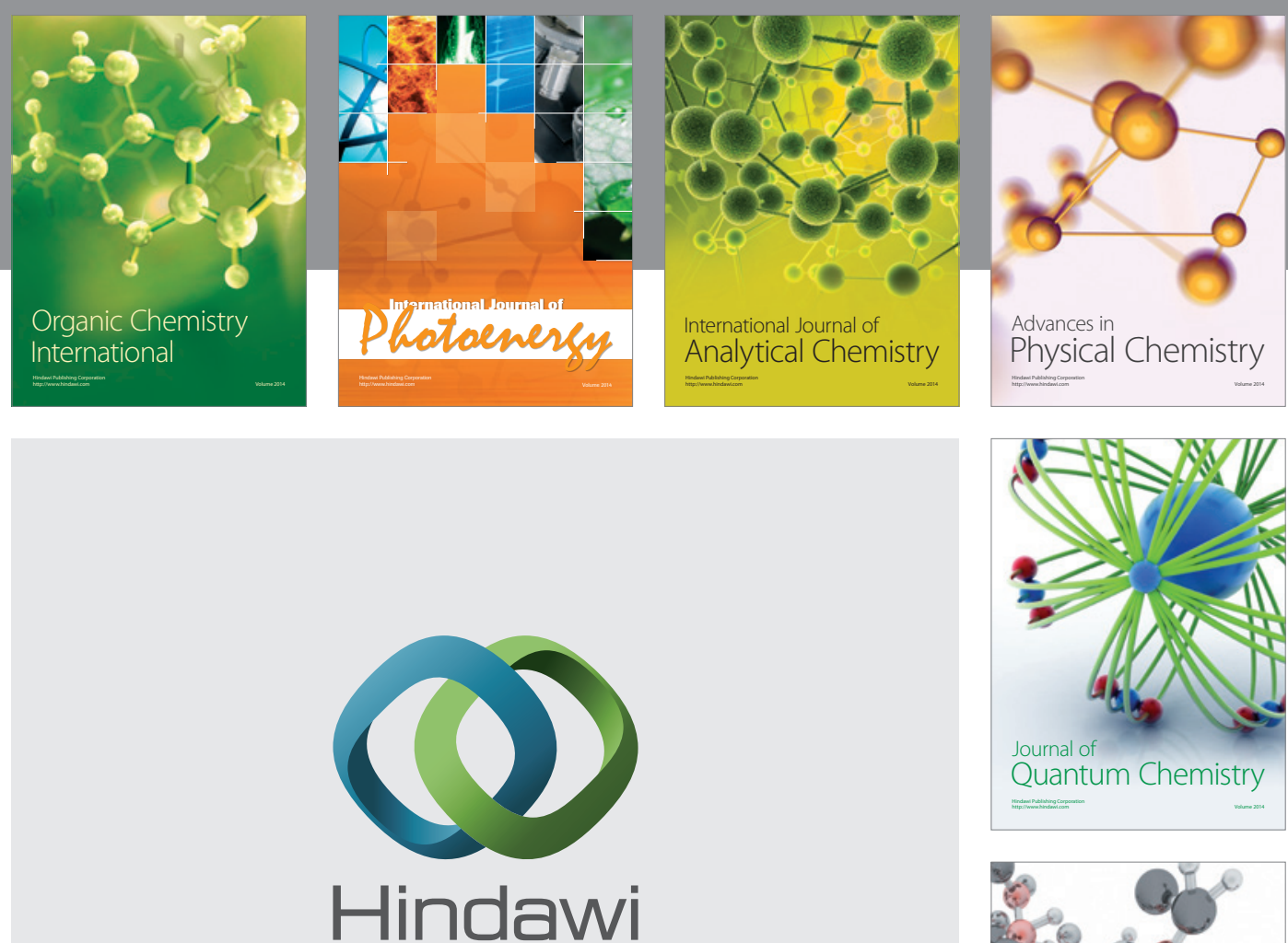

Submit your manuscripts at

http://www.hindawi.com

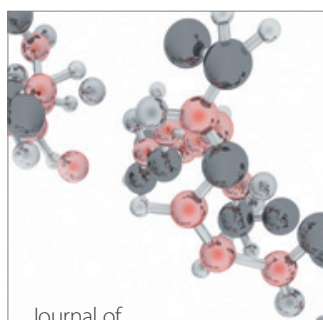

Analytical Methods

in Chemistry

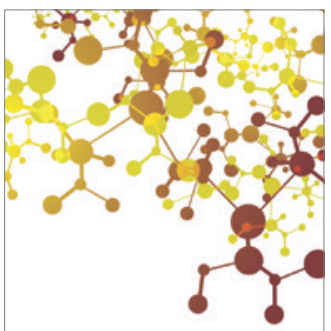

Journal of

Applied Chemistry

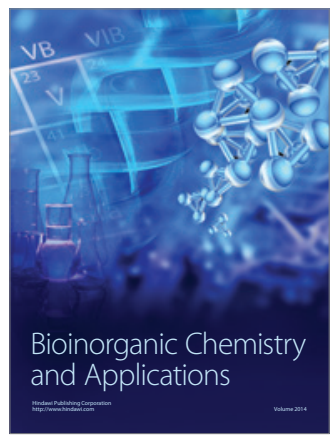

Inorganic Chemistry
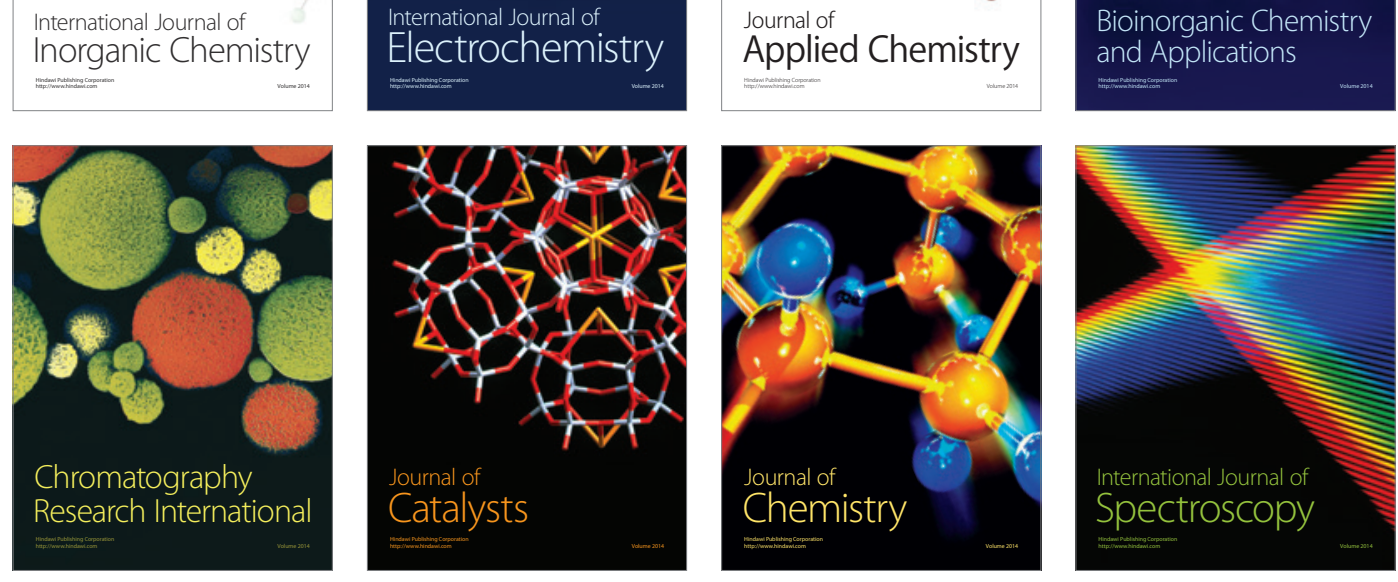